\title{
Complex dynamics and multistability with increasing rationality in market games
}

\author{
Fausto Cavalli ${ }^{\mathrm{b}, *}$, Ahmad Naimzada ${ }^{\mathrm{a}}$ \\ ${ }^{a}$ Dept. of Economics, Management and Statistics, University of Milano-Bicocca, U6 \\ Building, Piazza dell'Ateneo Nuovo 1, 20126 Milano, Italy \\ ${ }^{b}$ Department of Mathematical Sciences, Mathematical Finance and Econometrics, Catholic \\ University of the Sacred Heart, Via Necchi 9, 20123 Milano, Italy
}

\begin{abstract}
In this work we study oligopoly models in which firms adopt decision mechanisms based on best response techniques with different rationality degrees. Firms are also assumed to face resource or financial constraints in adjusting their production levels, so that, from time to time, they can only increase or decrease their strategy by a bounded quantity. We consider different families of oligopolies of generic sizes, characterized by heterogeneous compositions with respect to the rationality degrees of firms. We analytically study the local stability of the equilibrium depending on the oligopoly size and composition and through numerical simulations we investigate the possible dynamics arising when trajectories do not converge toward the equilibrium. We show that in this case complex dynamics can arise, and this is due to both the loss of stability of the equilibrium and to the emergence of multiple attractors, with the stable steady state coexisting with a different, periodic or chaotic, attractor. In particular, we show that multistability phenomena occur when the overall degree of rationality of the oligopoly is increased. Finally, we investigate the effect of non-convergent dynamics on the realized profits.
\end{abstract}

Keywords: Market games, bounded rationality, best response mechanisms, bifurcation, chaos, multistability.

\section{Introduction}

Due to the complexity of an oligopolistic market setting, firms should be more realistically assimilated to reduced rationality players, which only have a partial knowledge of the market and of their competitors' strategies. In classical market games, firms can compete with respect to the price of the good (Bertrand oligopoly [1]) or to the quantity to produce (Cournot oligopoly [2]).

\footnotetext{
* Corresponding author

Email addresses: fausto.cavalli@unicatt.it (Fausto Cavalli), ahmad.naimzada@unimib.it (Ahmad Naimzada)
} 
In latter case, under the assumption of bounded rationality, firms can only try to dynamically adapt their production levels toward the profit maximizing strategy, in a sort of evolutionary approximation of the Nash equilibrium. In the last twenty five years a very wide research strand has focused on the study of the dynamical properties of models describing market games among boundedly rational firms (for an in-depth review about possible decision mechanisms and oligopoly modelling, we refer to the book of Bischi et al. [3]). In particular, the dynamical adjustment of production levels may not converge toward the Nash equilibrium [2], following periodic, quasi-periodic and chaotic dynamics. Among the reasons for the occurrence of complex dynamics, we can mention the boundedly rational nature of the firms, the presence of nonlinearities in the market functions, behavioral or technological heterogeneities among firms and the oligopoly size. Restricting to the most recent works, we can mention the contributions about heterogeneous duopolies $[4,5,6,7,8]$, oligopolies of fixed, small sizes $[9,10,11,12]$ and oligopolies of generic sizes, with both fixed [13] and variable compositions $[14,15,13,16,17]$.

In the present work, we consider families of oligopolies of generic size $N$ obtained from heterogeneous combinations of three kinds of players, who have in common the decision mechanism, which is based on best response techniques, but who differ in their respective degrees of rationality. Firstly, for each model, we study the local stability of the steady state, which coincides with the Nash equilibrium. We analytically show for which oligopoly compositions and sizes the equilibrium is locally asymptotically stable. In particular, we prove that, for a suitably large fraction of rational players, we can have heterogeneous oligopolies whose equilibrium remains stable as their size increases. We then investigate through simulations the occurrence of complex dynamics. We show that when the equilibrium loses its stability, periodic and chaotic output trajectories can arise. More significantly, we show that even if the equilibrium is locally stable, we can have trajectories that converge toward a different attractor. Usually, multistability in the oligopolistic literature is connected to the technological heterogeneity of firms [5]. To the best of our knowledge, this is the first example of coexistence between differently complex attractors in oligopolies of generic sizes in which the only heterogeneity among firms concerns the rationality degree. In particular, we show that such level of complexity occurs increasing the overall rationality degree of the oligopoly, namely the fraction of rational players. This means that rational players can actually have a destabilizing effect, in the sense that the globally stable equilibrium can become just locally stable increasing their number.

Finally, we investigate the average profits achieved by each different kind of firms, in particular when trajectories do not converge toward the equilibrium. We show that, in such cases, realized profits are larger than equilibrium profits, so that instability can end in an advantage for firms. Moreover, especially when non-converging dynamics are due to multistability phenomena, it is no longer true that more rationality means larger profits.

The remainder of the paper is organized as follows. In Section 2, after presenting the economic setting, we introduce and describe LMA, Nash and rational 
players. In Section 3, we present four families of heterogeneous oligopolies and we analytically study the local stability of the equilibrium. In Section 4, we present numerical investigations. Conclusions and possible future researches are reported in Section 5.

\section{Cournot market games}

Let us consider an industry consisting of $N$ firms, indexed by $i=1, \ldots, N$, which produce quantities $q^{i}$ of homogeneous goods and have linear cost functions $C\left(q^{i}\right)=c q^{i}$, where $c>0$ represents the identical, constant marginal cost of each oligopolist. We assume that the price of the goods depends on the total output $Q$ through the isoelastic inverse demand function $p(Q)=1 / Q$ (more details can be found in $[2])$.

The profit of the $i$ th firm is then

$$
\pi_{i}\left(q^{i}, Q^{-i}\right)=\frac{q^{i}}{q^{i}+Q^{-i}}-c q^{i}
$$

where $Q^{-i}$ indicates the aggregate output level of all the oligopolists but the $i$ th one. Without loss of generality, from now on we can assume $c=1$. We notice that this means that all the analytical results presented in this section and the qualitative behavior of the numerical simulations reported in Section 4 are independent of $c$, which just rescales trajectories (setting $c=1$ actually corresponds to the change of variable $q^{i}:=c q^{i}$.) The previous framework sets up a game, in which players are the $N$ oligopolists, the set of admissible strategies consists of positive production levels $q^{i}>0$ and payoff functions are profit functions (1). Ahmed and Agiza [20] and Matsumoto and Szidarovski [21] showed that such game has only one Nash equilibrium $\left(q^{*}, \ldots, q^{*}\right) \in \mathbb{R}^{N}$ with

$$
q^{*}=\frac{N-1}{N^{2}}
$$

while the equilibrium aggregated quantity $Q^{*}$, profit $\pi^{*}$ and price $p^{*}$ are

$$
Q^{*}=\frac{N-1}{N}, \quad \pi^{*}=\frac{1}{N^{2}}, \quad p^{*}=\frac{N}{N-1} .
$$

In the oligopolies we aim to study, all the agents, who make their production choices at each discrete time $t \in \mathbb{N}$, adopt decision mechanisms based on best response techniques and differ in their rationality degrees, in particular with respect to the informational endowment. This means that some kinds of players have to adapt their production decisions at each time step, accordingly to an adjustment mechanism which depends on their, possibly reduced, informational endowment and on the, possibly variable, output levels of their competitors. This means that the difference $\left|\tilde{q}_{t+1}-q_{t}\right|$ between the next period output level $\tilde{q}_{t+1}$ given by the adjustment rule they adopt and the current production decision $q_{t}$ may be very large. However, in real situations, firms can meet constraints in trying to adapt their production levels. For example, they may be not able 
to immediately modify their output decision of any quantity. This is in general due to capacity and financial constraints or shortage of manpower, which prevent increasing the production level arbitrarily, as well as for economies of scale and break-even considerations or the impossibility to dismiss labor force, which do not allow firms to excessively decrease output decisions. A discussion about firms' behavior can be found in the book by Sterman [22]. For the reasons given above, it is more suitable to assume that firms gradually adapt their strategies toward the production level $\tilde{q}_{t+1}$. This can be modeled through the following adjustment mechanism

$$
q_{t+1}=q_{t}+\sigma\left(\tilde{q}_{t+1}-q_{t}\right)
$$

where $\sigma: \mathbb{R} \rightarrow \mathbb{R}$ is a differentiable, strictly increasing and bounded function which describes the feasible production variation. We assume $\sigma(0)=0$, so that steady states of the original adjustment mechanism are preserved and $\sigma^{\prime}(0)=1$. As we will see, this last assumption ensures that the local stability of the steady state is not affected by the particular choice of $\sigma$. Finally, we impose that $\sigma(x)$ is convex for $x<0$ and concave for $x>0$. This, together with the previous requirements, means that the more $\tilde{q}_{t+1}$ is different from $q_{t}$, the more $q_{t+1}$ is different from $\tilde{q}_{t+1}$. This allows mimicking that the output level adjustment is increasingly more difficult to realize as $\left|\tilde{q}_{t+1}-q_{t}\right|$ becomes large. We notice that similar gradual adjustment mechanisms have been proposed and used in oligopoly modelling $[23,15,13]$. Finally, we stress that it is possible to show that if no output limiter was considered, the players we are going to consider would choose to produce constantly null production levels for any sufficiently large oligopoly size (actually, approximatively $N>9$ ), which would mean that all firms would leave the market, which is indeed unrealistic.

To complete the description of the market game, we need to precise the assumptions on each kind of player. Firstly, we assume that players characterized by the same informational endowment are identical. In particular, this means that the starting production choice of such players is the same, and, consequently, production choices of such players coincide at each time. Moreover, each firm is supposed to know the oligopoly composition and the oligopoly size $N$, which are both constant in time. In what follows we consider three different kinds of players: local monopolistic approximation (LMA), Nash and rational players. LMA players adopts the so-called local monopolistic approximation, which is a boundedly rational mechanism, proposed and studied by Bischi et al. [19]. LMA firms are assumed to have a reduced informational endowment: they only know the current market price, their own current production quantity and they have a local knowledge of the demand function at the current price, obtained, for example, through market experiments. Nash players have complete information about the price function and the cost functions and make the assumption that all the other players have perfect foresight and use a best response mechanism. Moreover, they have enough computational capabilities to compute the Nash equilibrium (2). Finally, rational players know the demand function, the cost functions of each player and are able to compute the optimal 
output level which maximizes their profits with respect to the expected strategies of the other players. In particular, we assume that rational players are able to exactly foresight the production decisions that LMA and Nash players will adopt for the next period.

The remainder of this Section is devoted to the description of the discrete equations governing the adjustment mechanisms of each players. We notice that each firm is assumed to know the oligopoly composition and the oligopoly size $N$, which are both constant in time.

The adjustment mechanism of each LMA firm [24, 25] is given by

$$
\tilde{q}_{t+1}^{L}=\frac{1}{2} q_{t}^{L}+\frac{1}{2}\left(1-Q_{t}\right) Q_{t}
$$

where $Q_{t}$ is the aggregated quantity of all firms.

For the above mentioned reasons, we suppose that LMA players gradually adjust their strategy toward $\tilde{q}_{t+1}^{L}$, following (4). Introducing function $\lambda:\left(\mathbb{R}^{+}\right)^{2} \rightarrow \mathbb{R}$, defined by

$$
\lambda\left(q_{t}^{L}, Q_{t}^{-L}\right)=q_{t}^{L}+\sigma\left(\frac{1}{2} q_{t}^{L}+\frac{1}{2}\left(1-\left(\omega_{L} N q_{t}^{L}+Q_{t}^{-L}\right)\right)\left(\omega_{L} N q_{t}^{L}+Q_{t}^{-L}\right)-q_{t}^{L}\right)
$$

where $Q_{t}^{-L}$ is the aggregated quantity of non-LMA players, we obtain the adjustment mechanism of LMA players

$$
q_{t+1}^{L}=\lambda\left(q_{t}^{L}, Q_{t}^{-L}\right) .
$$

We notice that we may have $q_{t+1}^{L} \leq 0$. Since we aim to investigate the economically significant trajectories consisting of strictly positive output levels, we implicitly restrict ourselves to those initial conditions that guarantee $q_{t}^{L}>0$ for any $t>0$. Otherwise, in equation (7), we must take the maximum between the r.h.s. and 0 .

Regarding Nash players, since they figure that all players behave similarly to them, they compute the output level that each firm should play in order to optimize its profits, namely the Nash equilibrium strategy (2). We then have

$$
q_{t+1}^{N}=q^{*}=\frac{N-1}{N^{2}} .
$$

Finally, we need to specify the adjustment mechanism for rational players. Indeed, if the oligopoly only consisted of rational players, they would choose the Nash equilibrium strategy $q^{*}$ at any $t$. If there is at least a non-rational player, the best response of rational players depends on the strategies of the reduced rationality players. Such problem was studied by Cavalli et al. [13], who showed that the optimal production level of a generic rational player can be expressed in terms of the aggregated strategy $Q_{t+1}^{-R}$ of non-rational players, and results

$$
\tilde{q}_{t+1}^{R}=\frac{1-2 Q_{t+1}^{-R}}{2 \omega_{R} N}+\frac{\sqrt{\left(\omega_{R} N-1\right)^{2}+4 \omega_{R} N Q_{t+1}^{-R}}-1}{2 \omega_{R}^{2} N^{2}} .
$$


As for LMA players, we assume that rational players are not able to arbitrarily adapt their strategies from time $t$ to time $t+1$. In order to confine the heterogeneity among agents to their rationality degree, we suppose that rational players adopt the same output limiter of LMA players, so that

$q_{t+1}^{R}=\rho\left(q_{t}^{R}, Q_{t+1}^{-R}\right)=q_{t}^{R}+\sigma\left(\frac{1-2 Q_{t+1}^{-R}}{2 \omega_{R} N}+\frac{\sqrt{\left(\omega_{R} N-1\right)^{2}+4 \omega_{R} N Q_{t+1}^{-R}}-1}{2 \omega_{R}^{2} N^{2}}-q_{t}^{R}\right)$

where we introduced function $\rho:\left(\mathbb{R}^{+}\right)^{2} \rightarrow \mathbb{R}$. We remark that also in this case we only consider initial conditions that guarantee $q_{t}^{R}>0$ for any $t>0$.

\section{Analysis}

In this section we take into account families of oligopoly models which are heterogeneous with respect to the composition, namely in which at least two agents have different rationality degrees. We mainly focus on the analytical investigation of the local stability of steady states, depending on oligopoly size $N$ and composition. In what follows, we respectively identify the fraction of LMA, Nash and rational players with $\omega_{L}, \omega_{N}$ and $\omega_{R}$, through which the oligopoly composition is represented. We remark that, in the resulting models, both $\omega_{i}$ and $N$ are economically significant only if $\omega_{i}=k / N, k \in[0, N]$ and if $N \in \mathbb{N}$.

\subsection{Nash versus rational}

The first oligopoly family we consider is composed by heterogeneous combinations of Nash (8) and rational players (10). In what follows, we refer to it as NR. Since we aim at studying heterogeneous oligopolies, we have to impose that there are respectively at least a Nash and a rational firm, i.e.

$$
N \geq 2, \omega_{R} \in\left[\frac{1}{N}, 1-\frac{1}{N}\right]
$$

so that the fraction of Nash firms $\omega_{N}=1-\omega_{L}$ belongs to $[1 / N, 1-1 / N]$, too. The resulting NR model can be obtained from (10), in which the aggregated output level of non-rational players (namely, of Nash players) is $Q_{t}^{-R}=(1-$ $\left.\omega_{R}\right)(N-1) / N$. Inserting $Q_{t}^{-R}$ in $(10)$ we obtain map $f_{N R}: \mathbb{R}^{+} \rightarrow \mathbb{R}$ defined by

$$
q_{t+1}^{R}=f_{N R}\left(q_{t}^{R}\right)=\rho\left(q_{t}^{R},\left(1-\omega_{R}\right) \frac{N-1}{N}\right) .
$$

A straightforward computation shows that the unique steady state of (11) is $q^{*}$, which coincides with each component (2) of the Nash equilibrium. For NR model, we can prove that the steady state is globally stable.

Proposition 1. Steady state $q^{*}$ of $(11)$ is globally stable independently of the oligopoly size $N$ and composition $\omega_{R}$. 
Proof. It is easy to see that

$$
f_{N R}^{\prime}(q)=1-\sigma^{\prime}\left(-\frac{q N^{2}-N+1}{N^{2}}\right),
$$

from which we have $f_{N R}^{\prime}\left(q^{*}\right)=0$, which guarantees local stability. Moreover, since from the concavity/convexity assumptions on $\sigma$ we have that $\sigma^{\prime}(q)$ attains its maximum value 1 uniquely for $q=0$, we have that $f_{N R}^{\prime} \geq 0$. This allows concluding.

We notice that if rational players were able to immediately adjust their production level toward the best response strategy, in NR oligopolies each player would choose quantity $q^{*}$ in one shot. The only effect of introducing $\sigma$ is then to replace the instantaneous choice of $q^{*}$ with a gradual adjustment toward it, which however does not affect stability and convergence. Finally, we observe that the more bounds of function $\sigma$ are small (namely when rational firms can adapt their production level by a reduced quantity), the more convergence speed toward the equilibrium becomes slow.

\subsection{LMA versus Nash}

The second family of oligopolies we consider consists of heterogeneous combinations of LMA (7) and Nash (8) firms. We will refer to this model as LN. The heterogeneity assumption requires that there are at least a Nash and a LMA firm, namely

$$
N \geq 2, \omega_{L} \in\left[\frac{1}{N}, 1-\frac{1}{N}\right],
$$

so that the fraction of Nash firms $\omega_{N}=1-\omega_{L}$ belongs to $[1 / N, 1-1 / N]$, too. We have that the aggregated output level of non-LMA players is then $Q_{t}^{-L}=$ $\left(1-\omega_{L}\right)(N-1) / N$, which inserted into (7) allows defining map $f_{L N}: \mathbb{R}^{+} \rightarrow \mathbb{R}$ as

$$
q_{t+1}^{L}=f_{L N}\left(q_{t}^{L}\right)=\lambda\left(q_{t}^{L},\left(1-\omega_{L}\right) \frac{N-1}{N}\right),
$$

which represents LN model. It is easy to prove that the unique steady state of (13) is $q^{*}$, which coincides with each component (2) of the Nash equilibrium. The local stability of $q^{*}$ is studied in the next Proposition.

Proposition 2. Steady state $q^{*}$ of (13) is locally asymptotically stable provided that

$$
\left(\frac{N}{2}-1\right) w_{L}<\frac{3}{2}
$$

Proof. The proof is straightforward. It is sufficient to notice that $q^{*}$ is locally asymptotically stable provided that $\left|f_{L N}^{\prime}\left(q^{*}\right)\right|<1$. Since $f_{L N}^{\prime}\left(q^{*}\right)=(1-N / 2) \omega_{L}+$ $1 / 2$, we indeed have that $f_{L N}^{\prime}\left(q^{*}\right)<1$ is always fulfilled, while solving $f_{L N}^{\prime}\left(q^{*}\right)>$ -1 provides (14). 
We notice that, as $N$ increases, condition (14) is fulfilled by increasingly small values of $\omega_{L}$, namely the steady state is stable only if the fraction of LMA players is suitably small. This also means that keeping (approximatively) fixed the fraction $\omega_{L}$ of LMA firms and increasing $N$, we always have a sufficiently large number of firms for which the equilibrium becomes unstable. In the next Corollary, we precise, depending on the oligopoly size, the oligopoly compositions with locally stable steady state.

Corollary 1. Concerning LN oligopolies, for any size $N$ there exists a heterogeneous composition consisting of a suitably large number of Nash firms for which the steady state is locally asymptotically stable. In particular

1) if $N=2,3,4,5$ then $q^{*}$ is locally stable for any heterogeneous oligopoly composition;

2) if $N=6,7$ then $q^{*}$ is locally stable provided that we have at most 4 LMA firms;

3) if $N \geq 8$ then $q^{*}$ is locally stable provided that we have no more than 3 LMA firms.

Proof. For the proof of case 1), we notice that (14) is fulfilled if $N=2$. Conversely, we can write $\omega_{L}<3 /(N-2)$. Under assumption (12), such inequality is satisfied by any $\omega_{L}$ if

$$
1-\frac{1}{N}<\frac{3}{N-2}
$$

or equivalently if $N^{2}-6 N+2<0$, which requires $0.35 \approx 3-\sqrt{7}<N<$ $3+\sqrt{7} \approx 5.64$. Since $1 / N<3 /(N+2)$, we have unconditional stability with respect to oligopoly composition $\omega_{L}$ also for $N=3,4,5$.

To prove cases 2) and 3), we need to find the largest integer $n$ that solves

$$
\omega_{L}=\frac{n}{N}<\frac{3}{N-2}
$$

or, equivalently, $n<3+6 /(N-2)$. For $N=5,6$ we have that $1<6 /(N-2)<2$, so $4<3+6 /(N-2)<5$ and this allows choosing $n=4$. Conversely, for $N \geq 8$ we have $3<3+6 /(N-2) \leq 4$, so $n=3$.

The previous considerations also prove that for any $N$ we can always find $n \leq 3$ for which the steady state is locally stable.

As $N$ increases, the maximum number of LMA firms for which $q^{*}$ is locally stable is reported in Figure 1. The results of the previous Corollary are quite interesting, especially if compared to those about homogeneous oligopolies. As proved by Bischi et al. [19], a homogeneous oligopoly consisting of LMA firms has locally asymptotically stable equilibrium only if it consists of at most $N=4$ LMA firms. However, Corollary 1 shows that even adding "unconditionally stable" Nash firms, the maximum number of LMA firms that preserve equilibrium stability is reduced to 3 for any $N \geq 5$. Moreover, even if for any $N$ we can 
always find a heterogeneous combination of Nash and LMA firms that assures local stability, the maximum fraction of LMA players that preserve it tends toward zero as $N$ increases.

\section{3. $L M A$ versus rational}

The third family of heterogeneous oligopolies is composed by LMA (7) and rational (10) firms, and we refer to it as LR. In this case we have

$$
N \geq 2, \omega_{L} \in\left[\frac{1}{N}, 1-\frac{1}{N}\right]
$$

so $\omega_{R}=1-\omega_{L} \in[1 / N, 1-1 / N]$. In the present setting non-LMA players are rational players and then their aggregated production is $Q_{t}^{-L}=\left(1-\omega_{L}\right) N q_{t}^{R}$, while concerning the aggregated quantity $Q_{t+1}^{-R}$ of non-rational players (i.e. of LMA players) we can write

$$
Q_{t+1}^{-R}=\omega_{L} N q_{t+1}^{L}=\omega_{L} N \lambda\left(q_{t}^{L},\left(1-\omega_{L}\right) N q_{t}^{R}\right) .
$$

Using $Q_{t}^{-L}$ and $Q_{t+1}^{-R}$ in (10) and in (7), we obtain map $f_{L R}:\left(\mathbb{R}^{+}\right)^{2} \rightarrow \mathbb{R}$ defined by

$$
\left\{\begin{array}{l}
q_{t+1}^{L}=\lambda\left(q_{t}^{L},\left(1-\omega_{L}\right) N q_{t}^{R}\right), \\
q_{t+1}^{R}=\rho\left(q_{t}^{R}, \omega_{L} N \lambda\left(q_{t}^{L},\left(1-\omega_{L}\right) N q_{t}^{R}\right)\right),
\end{array}\right.
$$

which describes model LR. It is easy to see that $\left(q^{*}, q^{*}\right)$ is the only strictly positive steady state for $(15)$.

Local stability of $\left(q^{*}, q^{*}\right)$ is studied in the next Proposition.

Proposition 3. Steady state $\left(q^{*}, q^{*}\right)$ is locally asymptotically stable provided that

$$
4(N-2) \omega_{L}-3(N-1)<0 .
$$

Proof. To prove stability, we evaluate at the steady state the Jacobian matrix $J\left(q^{*}, q^{*}\right)$ of system (15). Recalling that $\sigma(0)=0$ and $\sigma^{\prime}(0)=1$, long but easy computations show that we have $J\left(q^{*}, q^{*}\right)=\left(a_{i j}\right)_{i, j=1,2}$ with

$$
\begin{gathered}
a_{11}=\frac{\omega_{L}(N-2)^{2}\left(1-\omega_{L}\right)}{2 N+4 \omega_{L}-2 N \omega_{L}-2}, \\
a_{12}=-\frac{\omega_{L}(N-2)\left(2 \omega_{L}-N \omega_{L}+1\right)}{2 N+4 \omega_{L}-2 N \omega_{L}-2}, \\
a_{21}=\frac{(N-2)\left(\omega_{L}-1\right)}{2}, \quad a_{22}=1-\frac{N \omega_{L}-2 \omega_{L}+1}{2},
\end{gathered}
$$

from which we obtain $\operatorname{det}\left(J\left(q^{*}, q^{*}\right)\right)=0$ and

$$
\operatorname{tr}\left(J\left(q^{*}, q^{*}\right)\right)=1-\frac{N-1}{2\left(N+2 \omega_{L}-N \omega_{L}-1\right)} .
$$

Consequently, the eigenvalues of $J\left(q^{*}, q^{*}\right)$ are $\lambda_{1}=\operatorname{tr}\left(J\left(q^{*}, q^{*}\right)\right)$ and $\lambda_{2}=0$. Stability condition then reduces to $\left|\lambda_{1}\right|<1$. Since $2\left(N+2 \omega_{L}-N \omega_{L}-1\right)>0$, we have $\lambda_{1}<1$, while imposing $\lambda_{1}>-1$ leads to (16). 
Condition (16) says that local stability is guaranteed only if the fraction of LMA firms is sufficiently small. However, differently from condition (14), we do not necessarily have that increasing $N$ and keeping $\omega_{L}$ (approximatively) fixed leads to instability. The precise number of rational firms needed to guarantee local stability is studied in the next Corollary. We recall that $\lceil a\rceil$ stands for the smallest integer greater or equal than $a$.

Corollary 2. Concerning LR oligopolies, for any size $N$ there exists a heterogeneous composition consisting of a suitably large number of rational firms for which the steady state is locally asymptotically stable. In particular

1) if $N \leq 7$ then $\left(q^{*}, q^{*}\right)$ is locally stable for any heterogeneous oligopoly composition;

2) if $N \geq 8$ then $\left(q^{*}, q^{*}\right)$ is locally stable provided that we have at least $\lceil(N-$ 3)/47 rational firms.

Proof. To prove case 1), we start noticing that (16) is indeed satisfied if $N=2$, while for $N>2$ we can write

$$
\omega_{L}<\frac{3}{4}\left(1+\frac{1}{N-2}\right) .
$$

Observing that for $1<N<8$ we have

$$
1-\frac{1}{N}<\frac{3}{4}\left(1+\frac{1}{N-2}\right)
$$

allows concluding.

To prove case 2), let $n$ be the number of rational firms, so that $\omega_{L}=1-n / N$. We need to find the smallest integer $n$ that for $N \geq 8$ solves

$$
1-n / N<\frac{3}{4}\left(1+\frac{1}{N-2}\right)
$$

which is equivalent to

$$
n>z(N)=\frac{N-3}{4}-\frac{3}{2(N-2)} .
$$

We notice that for $N=8$ we have $z(N)=1$, so we need at least $n=2=$ $\lceil(N-3) / 4\rceil$. When $N>8$, we can write $N=4 m+r$, where $m \in \mathbb{N}$ and $r=0,1,2,3$. Since $3 /(2(N-2)) \leq 3 / 14$, we have

$$
\frac{4 m+r-3}{4}-\frac{3}{2(N-2)}>m+\frac{r}{4}-\frac{3}{4}-\frac{3}{14}>m-1,
$$

and

$$
\frac{4 m+r-3}{4}-\frac{3}{2(N-2)}<m+\frac{r}{4}-\frac{3}{4} \leq m .
$$

Noticing that $m=\lceil(N-3) / 4\rceil$ allows concluding.

The previous considerations guarantee that, for any $N$, there always exists a sufficiently large number of rational firms such that the steady state is stable. 
The main consequence of the previous Corollary is that there are configurations for which the steady state is locally stable independently of $N$. In fact, if the oligopoly consists of at least a quarter of rational firms, the equilibrium is stable for any $N$. Such result goes against the literature about homogeneous oligopolies [26], in which the number of firms always has a destabilizing role. To the best of our knowledge, this is the first example of oligopoly in which the equilibrium remains locally stable for any $N .{ }^{1}$ The maximum number of LMA firms for which the equilibrium is locally stable is shown in Figure 1.

\subsection{LMA versus rational and Nash}

In the last family of oligopolies each mechanism is adopted by at least one agent, requiring

$$
N \geq 3, \omega_{L} \in\left[\frac{1}{N}, 1-\frac{2}{N}\right], \omega_{R} \in\left[\frac{1}{N}, 1-\frac{2}{N}\right],
$$

so that $\omega_{N}=1-\omega_{L}-\omega_{R} \in[1 / N, 1-2 / N]$. This means that the aggregated quantity of non-LMA players is given by

$$
Q_{t}^{-L}=\frac{\left(1-\omega_{R}-\omega_{L}\right)(N-1)}{N}+\omega_{R} N q_{t}^{R},
$$

while that of non-rational players is

$$
\begin{aligned}
Q_{t+1}^{-R}= & \frac{\left(1-\omega_{R}-\omega_{L}\right)(N-1)}{N} \\
& +\omega_{L} N \lambda\left(q_{t}^{L}, \frac{\left(1-\omega_{R}-\omega_{L}\right)(N-1)}{N}+\omega_{R} N q_{t}^{R}\right) .
\end{aligned}
$$

Putting $Q_{t}^{-L}$ and $Q_{t+1}^{-R}$ in (10) and in (7) we can introduce function $f_{L R N}$ : $\left(\mathbb{R}^{+}\right)^{2} \rightarrow \mathbb{R}$ defined by

$$
\left\{\begin{aligned}
q_{t+1}^{L}= & \lambda\left(q_{t}^{L}, \frac{\left(1-\omega_{R}-\omega_{L}\right)(N-1)}{N}+\omega_{R} N q_{t}^{R}\right) \\
q_{t+1}^{R}= & \rho\left(q_{t}^{R}, \frac{\left(1-\omega_{R}-\omega_{L}\right)(N-1)}{N}\right. \\
& \left.+\omega_{L} N \lambda\left(q_{t}^{L}, \frac{\left(1-\omega_{R}-\omega_{L}\right)(N-1)}{N}+\omega_{R} N q_{t}^{R}\right)\right) .
\end{aligned}\right.
$$

System (18) represents the dynamical model of LMA versus rational and Nash market game. It is easy to see that $\left(q^{*}, q^{*}\right)$ is the only steady state for (18), whose local stability is studied in the next Proposition.

Proposition 4. Steady state $\left(q^{*}, q^{*}\right)$ is locally asymptotically stable provided that

$$
(N-2) \omega_{L}-3 \omega_{R}(N-2)-3<0 .
$$

\footnotetext{
${ }^{1}$ The only example of an oligopoly in which the steady state does not lose local stability as the number of firms increases has been proposed by Puu [27] for a homogeneous oligopoly, in which however capacity limits are incorporated in cost function.
} 
Proof. We proceed as in the proof of Proposition 3. We have that the Jacobian matrix of system (18) evaluated at the steady state is $J\left(q^{*}, q^{*}\right)=\left(a_{i j}\right)_{i, j=1,2}$, where

$$
\begin{gathered}
a_{11}=\frac{\omega_{L} \omega_{R}(N-2)^{2}}{2\left(N \omega_{R}-2 \omega_{R}+1\right)}, \\
a_{12}=-\frac{\omega_{L}(N-2)\left(2 \omega_{L}-N \omega_{L}+1\right)}{2 N \omega_{R}-4 \omega_{R}+2}, \\
a_{21}=-\frac{\omega_{R}(N-2)}{2}, \quad a_{22}=\omega_{L}-\frac{N \omega_{L}}{2}+\frac{1}{2} .
\end{gathered}
$$

From the expression of $J\left(q^{*}, q^{*}\right)$ we have $\operatorname{det}\left(J\left(q^{*}, q^{*}\right)\right)=0$ and

$$
\operatorname{tr}\left(J\left(q^{*}, q^{*}\right)\right)=\frac{1}{2}-\frac{\omega_{L}(N-2)}{2\left(N \omega_{R}-2 \omega_{R}+1\right)},
$$

from which the eigenvalues of $J\left(q^{*}, q^{*}\right)$ are $\lambda_{1}=\operatorname{tr}\left(J\left(q^{*}, q^{*}\right)\right)$ and $\lambda_{2}=0$. Imposing stability condition $\left|\lambda_{1}\right|<1$, after noticing that, under assumption (17), we have $\lambda_{1} \leq 1 / 2<1$, the only substantial stability condition is $\lambda_{1}>-1$, which, after some simple algebraic manipulations, provides (19).

Stability condition (19) depends on both the fractions of LMA players and Nash players. As predictable, the 1.h.s. of (19) increases as $\omega_{L}$ increases, which highlights the destabilizing role of LMA firms. Conversely, it decreases as $\omega_{R}$ increases, so that increasing the number of rational firms has a stabilizing effect on the steady state. More precisely, we have the following Corollary.

Corollary 3. Let us consider LRN oligopolies and suppose that we have $1 \leq$ $n \leq N-2$ LMA firms, $1 \leq m \leq N-n-1$ rational firms and, consequently, $1 \leq N-n-m \leq N-2$ Nash firms. Then for any oligopoly size, there exists a heterogeneous composition consisting of a suitably large number of rational firm for which the steady state is locally asymptotically stable. In particular

1) if $N \leq 8$ then $\left(q^{*}, q^{*}\right)$ is locally stable for any heterogeneous oligopoly composition;

2) if $N \geq 9$ and if there are no more than $n=6 \mathrm{LMA}$ firms, then $\left(q^{*}, q^{*}\right)$ is locally stable independently of the number of rational and Nash firms;

3) if $N \geq 9$ and there are $n \geq 7$ LMA firms, then $\left(q^{*}, q^{*}\right)$ is locally stable if and only if the number of rational firms is $m \geq n / 3-1$.

Proof. From (17) and (19), using $\omega_{L} \leq 1-2 / N$ and $\omega_{R} \geq 1 / N$ we have

$$
(N-2)\left(\omega_{L}-3 \omega_{R}\right)-3<\frac{N^{2}-10 N+10}{N},
$$

so that condition (19) is satisfied provided that $N^{2}-10 N+10<0$, which means $1.13 \approx 5-\sqrt{15}<N<\sqrt{15}+5 \approx 8.88$. This proves case 1$)$. 
To prove case 2 ), we notice that since $\omega_{R} \geq 1 / N$ we have

$$
(N-2)\left(\omega_{L}-3 \omega_{R}\right)-3<(N-2)\left(\omega_{L}-\frac{3}{N}\right)-3
$$

and then a sufficient condition for stability is

$$
\omega_{L}<3\left(\frac{1}{N}+\frac{1}{N-2}\right)
$$

We notice that (20) guarantees local stability independently of the partition of rational/Nash firms. Since $\omega_{L}=n / N$, we can rewrite (20) as

$$
n<3+\frac{3 N}{N-2}=6+\frac{6}{N-2}<6.86
$$

for $N \geq 9$, which provides case 2 ).

To prove case 3 ), we notice that (19) can be rewritten as

$$
\omega_{R}>\frac{\omega_{L}}{3}+\frac{1}{N-2},
$$

which, using $\omega_{L}=n / N$ and $\omega_{R}=m / N$, becomes

$$
m>\frac{n}{3}-1-\frac{2}{N-2}
$$

Since $2 /(N-2)<0.29$ for $N \geq 9$, the previous relation is satisfied if and only if $m \geq n / 3-1$. This concludes the proof.

The previous considerations show that for any $N$ there is always a suitable number of rational firms that guarantees steady state stability.

The steady state in LNR oligopoly is locally stable for a slightly reduced number of LMA players than LR oligopoly, as shown in Figure 1. However, a slightly reduced number of rational firms is sufficient to assure steady state stability for any oligopoly size. In fact, from case 3 ) of Corollary 3 , if we choose $n$ LMA firms and $m \geq n / 3-1$ rational firms, then equilibrium stability is guaranteed. Assuming that there is only one Nash firm, we have $N=n+m+1 \leq$ $4 m+4$, so that $m \geq\lceil(N-4) / 4\rceil$. We again have that a quarter of rational firms is sufficient to guarantee local stability.

\section{Complex dynamics and Multistability}

We now investigate through simulations the possible dynamics arising in LN, LR, LNR models. Firstly, in order to perform simulations, we need to specify an explicit expression for function $\sigma$. We choose

$$
\sigma(x)= \begin{cases}a_{1} \tanh \left(\frac{x}{a_{1}}\right) & x \geq 0 \\ a_{2} \tanh \left(\frac{x}{a_{2}}\right) & x<0\end{cases}
$$




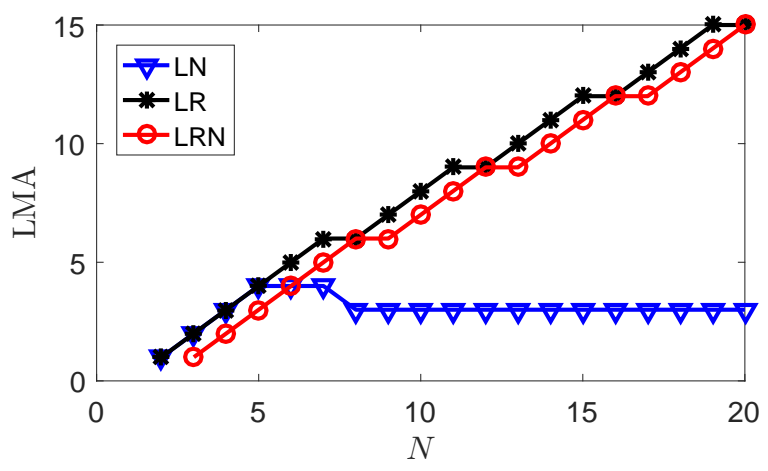

Figure 1: Plot of the maximum number of LMA firms for which we have a heterogeneous composition with a locally stable equilibrium. Blue plot refers to LN model (13), black plot refers to LR model (15) and red plot refers to LNR model (18).

where $-a_{2}$ and $a_{1}$ are respectively the lower and upper asymptotic bounds of function $\sigma$. It is easy to see that (21) fulfills all the requirements imposed on $\sigma$ in Section 2. We notice that the particular choice of function $\sigma$ does not significantly affect the resulting dynamics, as we numerically checked.

In order to obtain bifurcation diagrams on varying the oligopoly composition and size, we treat $\omega_{i}$ and $N$ as continuous variables. We also investigate the performance of each kind of mechanism, comparing the average profits

$$
m(\pi)=\frac{1}{t_{b}-t_{a}} \sum_{t=t_{a}}^{t_{b}} \pi_{t}
$$

achieved by each kind of firm in the different families of oligopolies. Indeed, we are interested in investigating those situations in which output trajectories do not converge toward the equilibrium, otherwise $m(\pi)$ would coincide with equilibrium profit $\pi^{*}$. In all the following simulations we set $t_{a}=1000$ and $t_{b}=1200$, which provided a reliable and robust approximation for $m(\pi)$. Finally, throughout this section, we set the lower and upper bounds of production variation to $a_{1}=0.01$ and $a_{2}=0.02$ and, in bifurcation diagrams with respect to $\omega_{i}$, we consider oligopolies consisting of $N=25$ firms. In the following bifurcation diagrams we only report variable $q^{L}$, since $q^{R}$ is qualitatively very similar.

We start investigating LN model. In Figure 2 we show the possible behavior of map (13), for some different oligopoly compositions, when the sigmoid function is (21). As we can see, the map, which lies between the two asymptotes $q_{L}-a_{1}$ and $q_{L}+a_{2}$, can be either increasing or bimodal. In depth numerical investigations suggest that, on varying $N$ and $\omega_{L}$, those are the only possible shapes of map (13).

In Figure 3 (a) we report the bifurcation diagram on varying $\omega_{L} \in[1 / N, 10 / N]$. Stability condition $(14)$ is $\omega_{L}<3 / 23 \approx 0.13$ and after this threshold a flip bifurcation occurs, with a subsequent cascade of period doublings leading to chaos. 


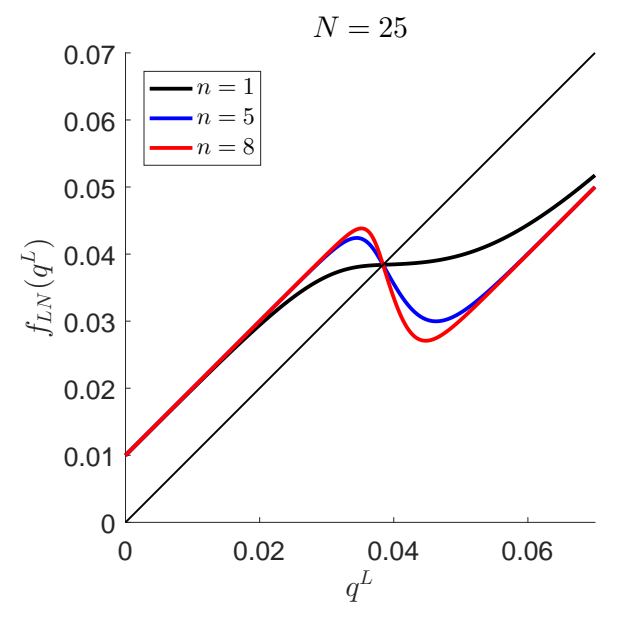

Figure 2: Map (13) for different numbers $n$ of LMA firms.

In particular, for $n \leq 3$ LMA firms the steady state is stable, for $n=4,5$ LMA firms we have a period- 2 cycle, for $n=6$ LMA firms we have a period- 4 cycle while for $n=7,8,9$ LMA firms the resulting dynamics are chaotic. In Figure 3 (a), the highlighted parts of the bifurcation diagram correspond to $n=2,5,6$ and 8 and display the increasing complexity. Finally, for $n>9$ LMA firms' dynamics are characterized by a period-3 cycle. In Figure 3 (b) we report the average profits realized by LMA and Nash firms for the same values of $\omega_{L}$ used for the bifurcation diagram in Figure 3 (a). Interestingly, when stability is lost, profits achieved by both kinds of firms are larger than equilibrium profits (3), with Nash firms which always achieve larger average profits. We numerically checked that both these results are robust with respect to the oligopoly size, and to the bounds of the output limiters, provided that the upper bound is smaller than that lower $\left(a_{2}>a_{1}\right)$. In the opposite situation $\left(a_{2}<a_{1}\right)$, profits achieved when equilibrium loses stability are smaller than equilibrium profits. However, Nash firms still achieve larger profits than LMA firms. In Figure 3 (c) we report the bifurcation diagram on varying $N \in[6,16]$, setting $\omega_{L}=1 / 2$. Also in this case, when stability condition $N<8$ is violated, we have a flip bifurcation ending with chaos. In Figure 3 (c) the cases of convergent dynamics $(N=6)$, of a 2-cycle $(N=10)$ and of chaotic dynamics $(N=16)$ are highlighted.

The next set of simulations concerns LR model. As we can see from the bifurcation diagram in Figure 4 (a), in this case different attractors coexist. While the simulations reported in Figure 3 were qualitatively independent of the particular initial datum, in this case we have that the resulting dynamics are strongly path-dependent. In Figure 4 (a), the black bifurcation diagram is obtained with an initial datum which is very close to the steady state, while the red diagram is obtained starting from $\left(q_{0}^{L}, q_{0}^{R}\right)=(0.1,0.02)$. Stability condition $(16)$ is $\omega_{L}<18 / 23 \approx 0.79$, but when another attractor emerges at $\omega_{L} \cong 0.562$, at which a saddle node bifurcation of a 2 -cycle occurs, leading an 


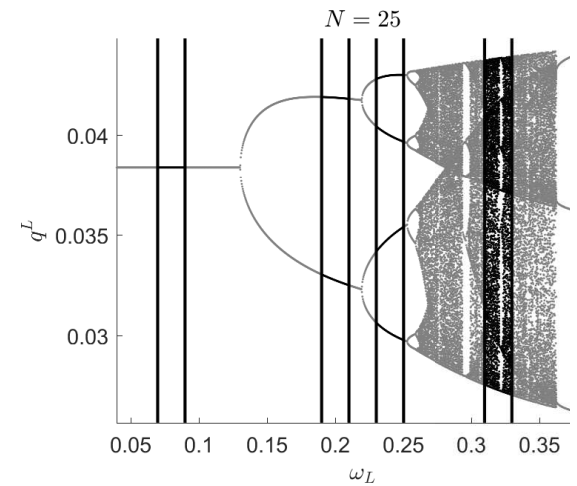

(a)

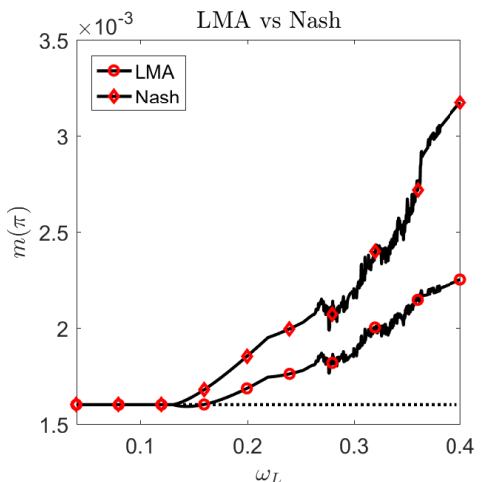

(b)

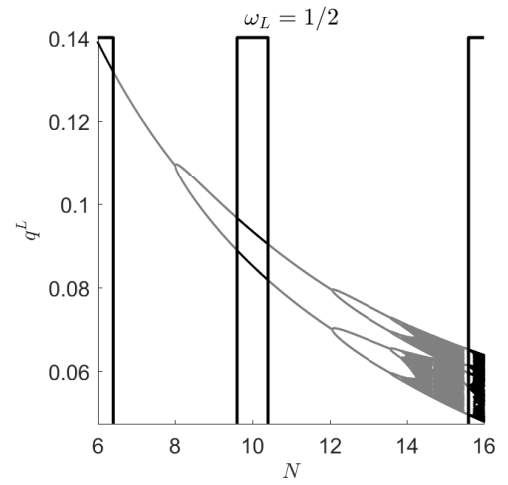

(c)

Figure 3: (a): Bifurcation diagram for LN model (13) as $\omega_{L}$ increases. Highlighted regions correspond to $n=2,5,6,8$ LMA firms.(b): Average profits achieved by LMA (circle) and Nash (diamond) firms, for the same values of $\omega_{L}$ used in bifurcation diagram (a). Red marks correspond to economically significant values of $\omega_{L}$. Equilibrium profits are represented by a dotted line. (c): Bifurcation diagram for LN model (13) as $N$ increases, for $\omega_{L}=0.5$. Highlighted regions correspond to oligopoly sizes $N=6,10,16$.

attracting 2-cycle to coexist with the attracting equilibrium point in the interval $\omega_{L} \in(0.562,0.71)$. In this case, as noticeable from the red bifurcation diagram in Figure 4 (a), dynamics initially consist of a period-2 cycle, which, as $\omega_{L}$ increases, undergoes a cascade of period doublings. Basins of attraction for $\omega_{L}=15 / N, 16 / N$ and $17 / N$, corresponding to the highlighted regions of Figure 4 (a), are reported in Figure 5, from which it is also evident that small perturbations of the equilibrium production can easily drive trajectories toward the coexisting attractor. If $\omega_{L}$ is increased above 0.71 the chaotic attractor collides, i.e. has a contact bifurcation, with the boundary of its basin, after which it become a chaotic repellor, and the equilibrium point is left as unique attractor. We stress that we thoroughly checked through numerical simulations that the steady state attracts almost all the points of the plane for $\omega_{L} \in(0.71,0.79)$. 


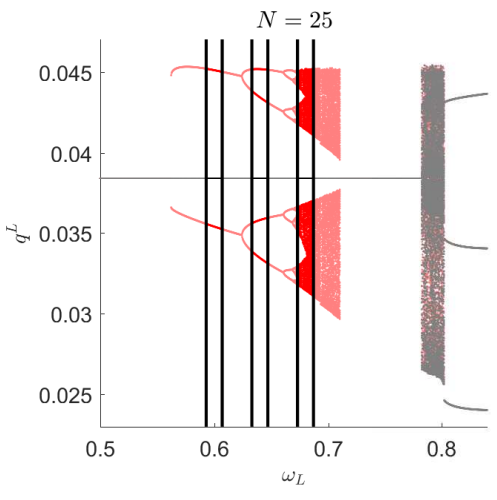

(a)

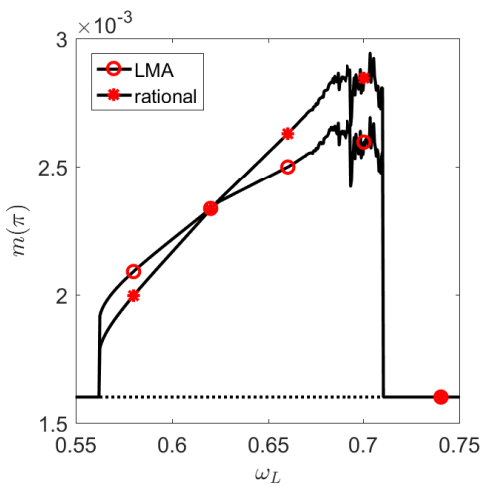

(b)

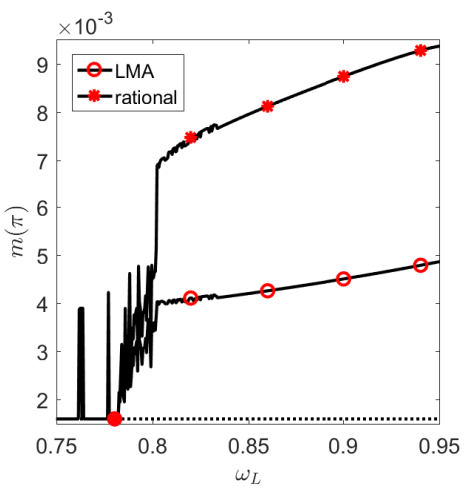

(c)

Figure 4: (a): Bifurcation diagrams for LR model (15) as $\omega_{L}$ increases. The black diagram is obtained for $\left(q_{0}^{L}, q_{0}^{R}\right) \approx\left(q_{0}^{*}, q_{0}^{*}\right)$, while the red diagram for $\left(q_{0}^{L}, q_{0}^{R}\right)=(0.1,0.02)$. Highlighted regions, in which different attractors coexist, correspond to $n=15,16,17$, LMA firms (b,c): Average profits, for $\omega_{L} \in(0.60 .77)$ (Figure (b)) and $\omega_{L} \in(0.790 .90)$ (Figure (c)), achieved by LMA (circle) and rational (star) firms, for the same values of $\omega_{L}$ used in bifurcation diagram (a). Red marks correspond to economically significant values of $\omega_{L}$. Equilibrium profits are represented by a dotted line. Plots (b) and (c) have different vertical scales.

When stability condition is violated, a flip bifurcation occurs, which however very soon disappears, replaced by a new chaotic attractor in one piece.

To the best of our knowledge, it is the first example of multistability with attractors of different complexity in heterogeneous oligopolies in which agents are homogeneous with respect to the technology. This is due to the presence of the more realistic bounded adjustment mechanism. The most interesting aspect is that in this scenario, rational players can actually have a destabilizing role. In fact, looking again at the bifurcation diagram in Figure 4 (a), if we consider oligopolies consisting of 18 and 19 LMA firms (i.e. $\omega_{L}=0.72,0.76$ ), the equilibrium attracts almost all the points of the plane. Conversely, if we consider 17 LMA firms (i.e. a larger number of rational firms), another attracting set with positive measure is present, and output decisions can follow a chaotic orbit. 
This means that replacing a reduced rationality firm with a rational firm can further the emergence of complex dynamics. We checked that the previous result is robust with respect to the oligopoly size, as we found a similar coexistence between different attractors for any $N \geq 20$.

We stress that LMA and rational firms only differ for the informational endowment and are identical with respect to marginal costs and output limiter. This result strengthens the findings of the work by Cavalli et al. [13], in which it was shown that increasing the number of rational players can introduce instability. However, in such paper, in which boundedly rational players adopted a best response mechanism with myopic expectations, to obtain a destabilizing effect for rationality, we had to take into account also technological heterogeneity, namely rational players had to face larger marginal costs than reduced rationality players.

We underline that the occurrence of multistability makes more difficult to understand the behavior of the profits achieved by each kind of firm. In Figure 4 (b) we report the average profits when production trajectories follow the coexisting attractor, for compositions for which the steady state is stable. As in LN model, when we are far from the steady state trajectory, firms achieve profits which are larger than equilibrium profits when $a_{2}>a_{1}$ (as in Figure 4 (b)) and which are smaller than equilibrium profits when $a_{1}>a_{2}$. However, in both cases, we also have situations in which the least rational firms (LMA) achieve larger profits. In Figure 4 (c) we collect the profits realized by LMA and rational firms when the steady state is unstable. In this case, rational firms' profits are larger than those of LMA firms.

Finally, we consider the family of oligopolies in which LMA, Nash and rational players are simultaneously present. In Figure 6 we show a two-dimensional bifurcation diagram with respect to $\omega_{L}$ and $\omega_{R}$, starting with initial condition $\left(q_{0}^{L}, q_{0}^{R}\right)=(0.195,0.195)$. The oligopoly compositions which provide a stable equilibrium correspond to the region described by (19), below the black curve of Figure 6. As we can see, we again have coexisting attractors, since in such region trajectories, which should actually converge toward the steady state (green color), converge toward different attractors consisting of periodic points and complex attractors (cyan region). This is also evident from the particular onedimensional bifurcation diagram reported in Figure 7 (a), obtained, as $\omega_{L}$ varies, for $\omega_{N}=3 / N$ and $\omega_{R}=1-\omega_{L}-\omega_{N}$. Red bifurcation diagram is obtained for the same initial condition used for the two-dimensional bifurcation diagram reported in Figure 6 while for the black diagram we set $\left(q_{0}^{L}, q_{0}^{R}\right) \approx\left(q_{0}^{*}, q_{0}^{*}\right)$. In the two highlighted regions of Figure 7 (a), corresponding to oligopolies in which $n=15$ and 16 LMA firms are considered, we respectively have a 2-cycle and a chaotic trajectory coexisting with the stable steady state. The previous example shows the potentially destabilizing role of rational players once more, as increasing their number opens the way for complex dynamics even in a context in which Nash players are present.

We remark that basins of attraction are qualitatively similar to those of LR model.

With respect to average profits, we can notice from Figures 7 (b) and (c) that 


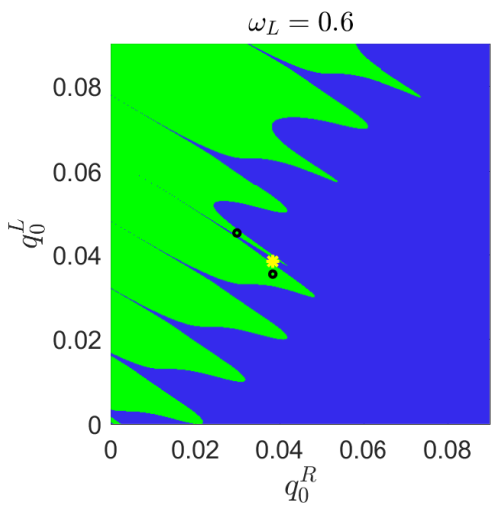

(a)

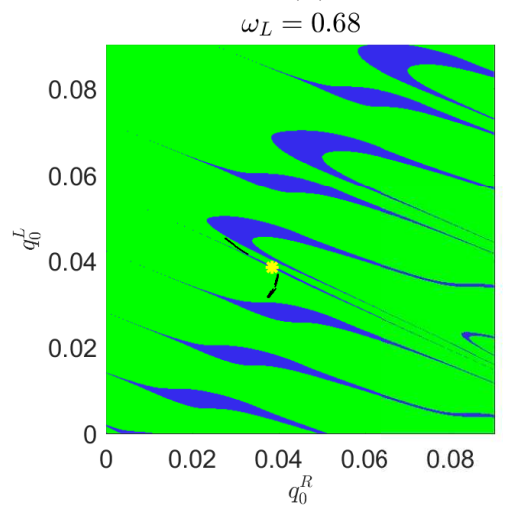

(c)

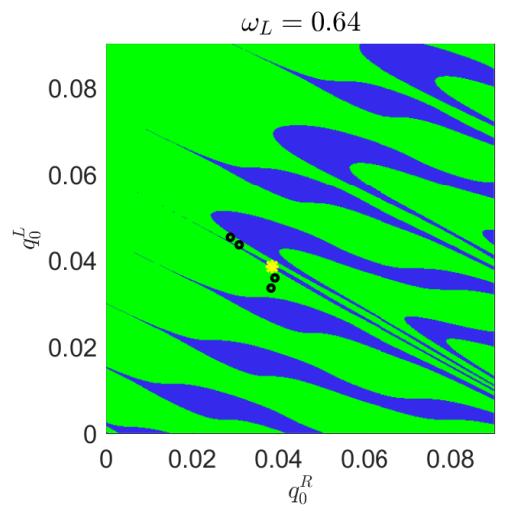

(b)

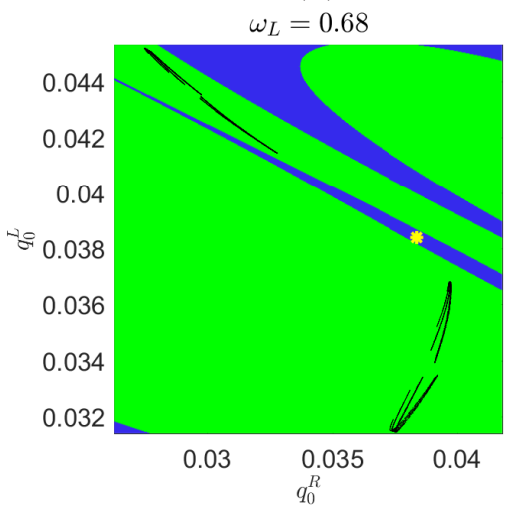

(d)

Figure 5: Basins of attraction for LR model (15), for three compositions related to the bifurcation diagram reported in Figure 4. Yellow star represents the equilibrium point, while black circles (Figures $(\mathrm{a}, \mathrm{b})$ ) and dots (Figures $(\mathrm{c}, \mathrm{d})$ ) represent the coexisting attractor. Blue color is used for the basin of the steady state, green color for that of the coexisting attractor. Figure (d) is an enlargement of Figure (c) in a neighborhood of the attractors.

we have both situations in which rational players realize the best performance and in which their average profits are the smallest one, being those of Nash players the largest ones.

\section{Conclusions}

In this work we studied oligopolies consisting of different heterogeneous combinations of players who adopt decision mechanisms based on best response techniques with different degrees of rationality. Moreover, to encompass in the model resource or financial constraints that prevent firms from being able to arbitrarily increase or decrease their output level, we considered a bounded adjustment mechanism. If the rationality degree is suitably elevated, as in the case of RN model, the presence of the output limiter does not affect stability 


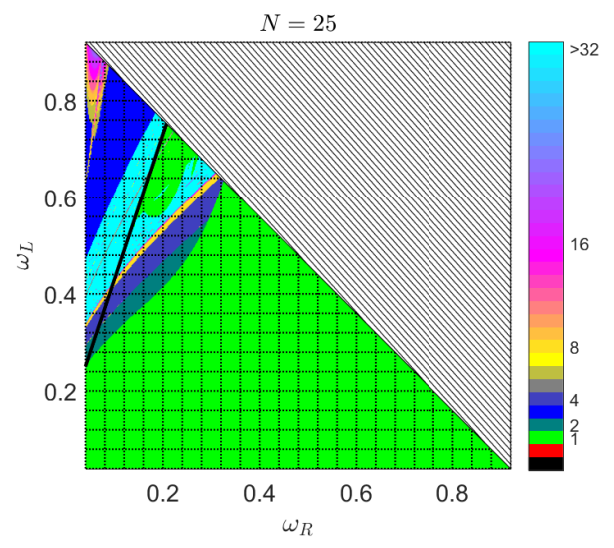

Figure 6: Two dimensional bifurcation diagram for LNR model (18) with respect to $\omega_{L}$ and $\omega_{R}$. Different colors are used to identify the number of points of the attractor set. The black line represents local stability threshold (19) below which the equilibrium is stable. At the intersections of dotted lines we have economically meaningful compositions. Hatched region consists of unfeasible compositions.

and convergence toward the equilibrium. However, we showed that it is possible that players with a high degree of rationality have a destabilizing effect, as increasing their number can destroy the global stability of the equilibrium, introducing the possibility of chaotic trajectories. As a consequence, we also showed that the performance in terms of profits of the firms can be no longer directly connected to their degree of rationality. This can make very difficult for the agents to refine their adjustment mechanisms on the base, for example, of the profits achieved by each kind of firm. The goal of the future research is to investigate, from an evolutionary point of view, the effect of an evolutive competition among the heuristics considered in the present work, in line with the seminal work by Brock and Hommes [28]. Moreover, since the learning process, which consists of the information collection about the economic environment, is usually carried out less frequently than production choices, we aim to study the effect of considering two different time scales for decision processes, as in the monopoly model considered by Cavalli and Naimzada [29].

\section{Acknowledgments}

The authors wish to thank the anonymous Referee for the helpful suggestions and comments.

[1] J. Ma, J. Zhang, Price game and chaos control among three oligarchs with different rationalities in property insurance market, Chaos 22 (2012) 043120 .

[2] T. Puu, Chaos in duopoly pricing, Chaos, Solitons and Fractals 1 (6) (1991) $573-581$. 


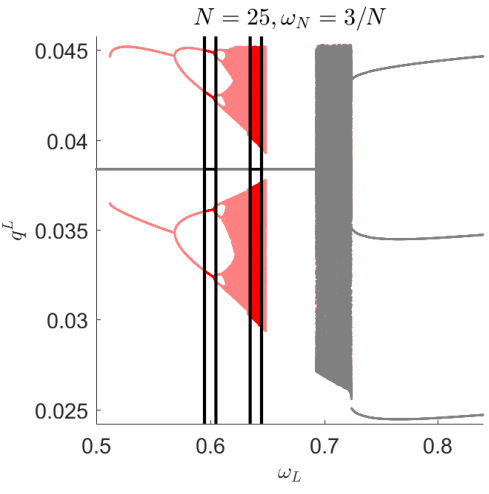

(a)

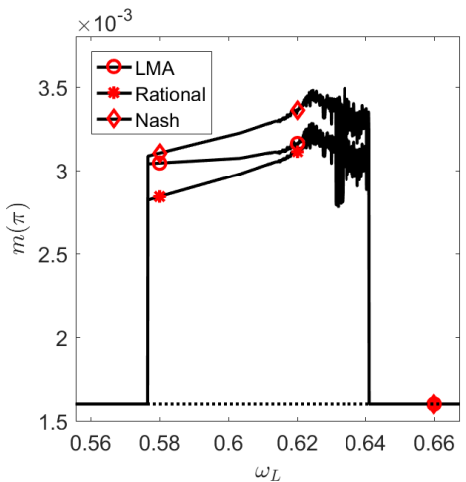

(b)

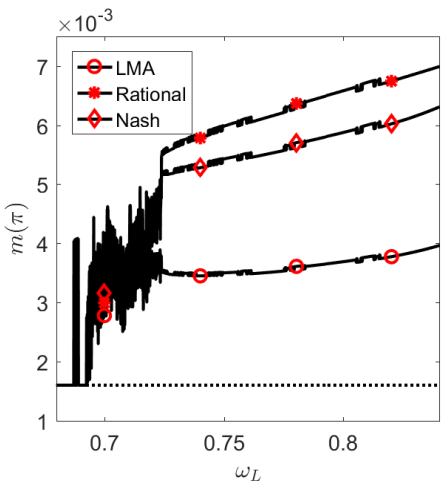

(c)

Figure 7: (a): Bifurcation diagrams for LNR model (18) as $\omega_{L}$ increases. The black diagram is obtained for $\left(q_{0}^{L}, q_{0}^{R}\right) \approx\left(q_{0}^{*}, q_{0}^{*}\right)$, while the red diagram for $\left(q_{0}^{L}, q_{0}^{R}\right)=(0.195,0.195)$. Highlighted regions correspond to $n=15,16$ LMA firms. (b,c): Average profits, for $\omega_{L} \in(0.590 .70)$ (Figure (b)) and $\omega_{L} \in(0.740 .85)$ (Figure (c)), achieved by LMA (circle), Nash (diamond) and rational (star) firms, for the same values of $\omega_{L}$ used in bifurcation diagram (a). Red marks correspond to economically significant values of $\omega_{L}$. Equilibrium profits are represented by a dotted line. Plots (b) and (c) have different vertical scales.

[3] G. I. Bischi, C. Chiarella, M. Kopel, F. Szidarowski, Nonlinear Oligopolies - Stability and Bifurcations, Springer, 2010.

[4] F. Cavalli, A. Naimzada, A Cournot duopoly game with heterogeneous players: nonlinear dynamics of the gradient rule versus local monopolistic approach, Applied Mathematics and Computation 249 (2014) 382-388.

[5] F. Cavalli, A. Naimzada, F. Tramontana, Nonlinear dynamics and global analysis of a heterogeneous Cournot duopoly with a local monopolistic approach versus a gradient rule with endogenous reactivity, Communications in Nonlinear Science and Numerical Simulation 23 (1-3) (2015) 245-262.

[6] F. Cavalli, A. Naimzada, Nonlinear dynamics and convergence speed of 
heterogeneous Cournot duopolies involving best response mechanisms with different degrees of rationality, Nonlinear Dynamics 81 (1) (2015) 967-979.

[7] L. Fanti, L. Gori, M. Sodini, Nonlinear dynamics in a Cournot duopoly with isoelastic demand, Mathematics and Computers in Simulation 108 (2015) 129-143.

[8] S. S. Askar, K. Alnowibet, Nonlinear oligopolistic game with isoelastic demand function: rationality and local monopolistic approximation, Chaos, Solitons and Fractals 84 (2016) 15-22.

[9] E. M. Elabbasy, H. N. Agiza, A. A. Elsadany, Analysis of nonlinear triopoly game with heterogeneous players, Computers and Mathematics with Applications 57 (2009) 488-499.

[10] A. A. Elsadany, Competition analysis of a triopoly game with bounded rationality, Chaos, Solitons and Fractals 45 (11) (2012) 1343 - 1348.

[11] A. A. Elsadany, H. N. Agiza, E. M. Elabbasy, Complex dynamics and chaos control of heterogeneous quadropoly game, Applied Mathematics and Computation 219 (2013) 11110-11118.

[12] F. Tramontana, A. A. Elsadany, B. Xin, H. N. Agiza, Local stability of the Cournot solution with increasing heterogeneous competitors, Nonlinear Analysis: Real World Applications 26 (2015) 150-160.

[13] F. Cavalli, A. Naimzada, M. Pireddu, Heterogeneity and the (de)stabilizing role of rationality, Chaos, Solitons and Fractals 79 (2015) 226-244.

[14] E. Droste, C. Hommes, J. Tuinstra, Endogenous fluctuations under evolutionary pressure in Cournot competition, Games and Economic Behavior 40 (2) (2002) 232-269.

[15] F. Cavalli, A. Naimzada, M. Pireddu, Effect of size, composition and evolutionary pressure in heterogeneous Cournot oligopolies with best response decisional mechanisms, Discrete Dynamics in Nature and Society 2015 (2015) 273026. doi:http://dx.doi.org/10.1155/2015/273026.

[16] G. I. Bischi, F. Lamantia, D. Radi, An evolutionary Cournot model with limited market knowledge, Journal of Economic Behavior and Organization 116 (2015) 219-238.

[17] L. Cerboni Baiardi, F. Lamantia, D. Radi, Evolutionary competition between boundedly rational behavioral rules in oligopoly games, Chaos, Solitons and Fractals 79 (2015) 204-225.

[18] J. Tuinstra, A price adjustment process in a model of monopolistic competition, International Game Theory Review 6 (3) (2004) 417-442. 
[19] G. I. Bischi, A. Naimzada, L. Sbragia, Oligopoly games with local monopolistic approximation, Journal of Economic Behavior and Organization 62 (3) (2007) 371-388.

[20] E. Ahmed, H. N. Agiza, Dynamics of a Cournot game with n-competitors, Chaos, Solitons and Fractals 9 (9) (1998) 1513-1517.

[21] A. Matsumoto, F. Szidarovszky, Stability, bifurcation, and chaos in N-firm nonlinear Cournot games, Discrete Dynamics in Nature and Society 2011 (2011) 380530. doi:http://dx.doi.org/10.1155/2011/380530.

[22] J. Sterman, Business Dynamics: Systems Thinking and Modeling for a Complex World, McGraw-Hill, 2000.

[23] J. G. Du, Y. Q. Fan, Z. H. Sheng, Y. Z. Hou, Dynamics analysis and chaos control of a duopoly game with heterogeneous players and output limiter, Economic Modelling 33 (2013) 507-516.

[24] A. Naimzada, F. Tramontana, Controlling chaos through local knowledge, Chaos, Solitons and Fractals 42 (4) (2009) 2439-2449.

[25] A. Naimzada, G. Ricchiuti, Monopoly with local knowledge of demand function, Economic Modelling 28 (1-2) (2011) 299-307.

[26] M. Lampart, Stability of the Cournot equilibrium for a Cournot oligopoly model with n competitors, Chaos, Solitons and Fractals 45 (9-10) (2012) 1081-1085.

[27] T. Puu, On the stability of Cournot equilibrium when the number of competitors increases, Journal of Economic Behavior and Organization 66 (3-4) (2008) 445-456.

[28] W. A. Brock, C. Hommes, A rational route to randomness, Econometrica 65 (5) (1997) 1059-1095.

[29] F. Cavalli, A. Naimzada, A multiscale time model with piecewise constant argument for a boundedly rational monopolist, Journal of Difference Equations and Applicationsdoi:10.1080/10236198.2016.1202940. 